DEPARTMENT OF ECONOMICS

Working Paper Series

Kuhn's Theorem for Extensive Games with Unawareness

Burkhard Schipper

University of California, Davis

November 02, 2017

Paper \# 17-6

We extend Kuhn's Theorem to extensive games with unawareness. This extension is not entirely obvious: First, extensive games with non-trivial unawareness involve a forest of partially ordered game trees rather than just one game tree. An information set at a history in one tree may consist of histories in a less expressive tree. Consequently, perfect recall takes a more complicated form as players may also become aware of new actions during the play. Second, strategies can only be partially an object of ex-ante choice in games with unawareness. Finally, histories that a player may expect to reach with a strategy profile may not be the histories that actually occur with this strategy profile, requiring us to define appropriate notions of equivalence of strategies.

Department of Economics

One Shields Avenue

Davis, CA 95616

(530)752-0741

http://wp.econ.ucdavis.edu/ 


\title{
Kuhn's Theorem for Extensive Games with UNAWARENESS*
}

\author{
Burkhard C. Schipper ${ }^{\dagger}$
}

November 1, 2017

\begin{abstract}
We extend Kuhn's Theorem to extensive games with unawareness. This extension is not entirely obvious: First, extensive games with non-trivial unawareness involve a forest of partially ordered game trees rather than just one game tree. An information set at a history in one tree may consist of histories in a less expressive tree. Consequently, perfect recall takes a more complicated form as players may also become aware of new actions during the play. Second, strategies can only be partially an object of ex-ante choice in games with unawareness. Finally, histories that a player may expect to reach with a strategy profile may not be the histories that actually occur with this strategy profile, requiring us to define appropriate notions of equivalence of strategies.
\end{abstract}

Keywords: Perfect recall, mixed strategy, behavior strategy, unawareness.

JEL-Classifications: C72, D83.

\footnotetext{
*I thank Aviad Heifetz and Martin Meier for many discussions about extensive games with unawareness. Past financial support through NSF SES-0647811 is gratefully acknowledged.

${ }^{\dagger}$ Department of Economics, University of California, Davis. Email: bcschipper@ucdavis.edu
} 


\section{Introduction}

In extensive games there are two notions of mixed strategies. First, a player can ex ante mix over her strategies, using a so called mixed strategy. Second, at each information set a player can mix interim over actions at that information set, using a so called behavior strategy. Kuhn's Theorem states that in every extensive game with perfect recall, for each mixed strategy there is a behavior strategy that is equivalent in terms of the probabilities of reaching nodes in the game tree (Kuhn, 1953, see also Selten, 1975). The use of mixed strategies have been questioned (see a discussion in Osborne and Rubinstein, 1994, Chapter 3.2). However, even if players do not actually play mixed strategies, players may ex ante form probabilistic beliefs over strategies of opponents, so called conjectures. Such a conjecture may be viewed as a mixed strategy. Similarly, a player may form beliefs over opponents' actions that they may play at each of their information sets, and a profile of such beliefs, one for each of the opponent's information set can be viewed as a behavior strategy. In any case, it is fair to say that Kuhn's Theorem is one of the most fundamental theorems on extensive games. It highlighted the assumption of perfect recall and facilitated the development of extensive game theory. Almost the entire literature on extensive games invokes the perfect recall assumption and often invokes in some form or another Kuhn's Theorem.

In extensive games with unawareness, the notion of mixed strategy is even less compelling. A player may not be aware of all actions ex ante. Thus, she may not be able to conceive of all possible strategies and hence may be unable to mix over them. Yet, given her awareness ex ante, she conceives of some partial strategies ex ante and be able to mixed over them. It begs now the question whether for each (partial) mixed strategy there is an equivalent (partial) behavior strategy. An immediate answer is clouded by several subtleties: First, extensive games with unawareness feature a forest of game trees rather than just one tree (see Halpern and Rêgo, 2014, Heifetz, Meier, and Schipper, 2013, Grant and Quiggin, 2013, Feinberg, 2012; see Schipper 2014 for a review). The relevant information set at a node in one tree may actually reside in a less expressive tree, signifying the fact that the player to whom this information set belongs to is unaware of something. These obvious differences in the formalism to standard extensive games make it non-trivial to define perfect recall in a meaningful way. Yet, the perfect recall assumption is instrumental for proving Kuhn's Theorem. In extensive games with unawareness, perfect recall interacts with other properties on how awareness differs across trees. In particular, these properties imply that a player's awareness may not decrease during

play. This property is also crucial for us proving the analogue of Kuhn's Theorem for extensive games with unawareness.

There is another challenge that Kuhn's Theorem faces in extensive games with unawareness. What is a meaningful notion of two strategies being equivalent to each other? Extensive games with unawareness allow the game theorist to model differing players' subjective conceptions of 
the game during the play. Since (partial) strategies are objects of players' beliefs, the notion of a strategy reaching a node or an information set is also subjective. A player may believe that a strategy reaches a particular node or a particular information set even though this node or information set cannot be realized with this strategy. We formalize these two notions of a strategy being consistent with a node or information set in an extensive games, show their relationship, and extend Kuhn's Theorem to both of these two notions in extensive games with unawareness.

Kuhn's Theorem for extensive games with unawareness is crucial for further developing games with unawareness. This paper is motivated by our work on self-confirming equilibrium in which we invoke the result to define we believe is a meaningful notion of equilibrium in games with unawareness, a notion that can be interpreted both as a steady state of conceptions and play (see Schipper, 2017). Extensive games with unawareness have been applied to political campaigning (Schipper and Woo, 2017), persuasion with verifiable disclosure (Heifetz, Meier, Schipper, 2012; see also Li and Schipper, 2017, for an experiment), and insurance contracts (Filiz-Ozbay, 2012). We have now all game theoretic tools ready to revisit games with incomplete information and explore how the presence of unawareness may change predictions.

We should state upfront that this paper has nothing to contribute to decision making under absentmindedness (see Piccione and Rubinstein, 1997, and the special issue on imperfect recall in Games and Economic Behavior 1997) beyond the fact that imperfect recall also implies absentmindedness in extensive games with unawareness. It is conceivable that in some circumstances, absentmindedness may be extreme in the sense of becoming unaware of some events that player had considered previously. Yet, becoming unaware of events is outside current models of extensive games with unawareness.

The paper is organized as follows: The next section spells out in detail extensive games with unawareness. Section 3 introduces various notions of strategies. Kuhn's Theorem is extended to extensive games with unawareness in Section 4.

\section{Extensive Games with Unawareness}

In this section, we outline extensive game with unawareness à la Heifetz, Meier, and Schipper (2013). ${ }^{1}$ To define an extensive game with unawareness $\Gamma$, consider first, as a building block,

\footnotetext{
${ }^{1}$ Although there are differences in the formalism between various approaches to extensive games with unawareness (Halpern and Rêgo, 2014, Heifetz, Meier, and Schipper, 2013, Grant and Quiggin, 2013, Feinberg, 2012, Rêgo and Halpern, 2012, Ozbay, 2007; see Schipper, 2014, for a brief review), all approaches model unawareness that is consistent with the paradigm of "propositional awareness" as in Fagin and Halpern (1988) or Heifetz, Meier, and Schipper (2006). We use here the approach by Heifetz, Meier, and Schipper (2013) because information sets in their approach can be interpreted as states of the mind of a player at a history. In their approach, information sets model both information and awareness rather than just "information if the player
} 
a finite game with perfect information and possibly simultaneous moves. The major purpose of this tree is to outline all physical moves. There is a finite set of players $I$ and possibly a special player "nature" with index 0 . We denote by $I^{0}$ the set of players including nature. Further, there is a nonempty finite set of "decision" nodes $\bar{D}$ and a player correspondence $P: \bar{D} \longrightarrow 2^{I^{0}} \backslash\{\emptyset\}$ that assigns to each node $n \in \bar{D}$, a nonempty set of "active" players $P(n) \subseteq I^{0}$. (That is, we allow for simultaneous moves as in Dubey and Kaneko, 1984, or in Osborne and Rubinstein, 1994, Chapter 6.3.2) For every decision node $n \in \bar{D}$ and player $i \in P(n)$ who moves at that decision node, there is a nonempty finite set of actions $A_{i}(n)$. Moreover, there is a set of terminal nodes $\bar{Z}$. Each terminal node $z \in \bar{Z}$ is associated with a vector of payoffs $\left(u_{i}(z)\right)_{i \in I}$, one for each player $i \in I$. We require that nodes in $\bar{N}:=\bar{D} \cup \bar{Z}$ constitute a tree denoted by $\bar{T}$. That is, nodes in $\bar{N}$ are partially ordered by a precedence relation $\lessdot$ with which $(\bar{N}, \lessdot)$ forms an arborescence (that is, the predecessors of each node in $\bar{N}$ are totally ordered by $\lessdot$ ). There is a unique node in $\bar{N}$ with no predecessors (i.e., the root of the tree). Finally, for each decision node $n \in \bar{D}$ there is a bijection $\psi_{n}$ between the action profiles $\prod_{i \in P(n)} A_{i}(n)$ at $n$ and $n$ 's immediate successors. Any terminal node in $\bar{Z}$ has no successors.

Note that so far we treat nature like any other player except that at terminal nodes we do not assign payoffs to nature. ${ }^{2}$ We do not need to require that nature moves first or that nature moves according to a pre-specified probability distribution (although these assumptions can be imposed in our framework).

Consider now a finite join-semilattice $\mathbf{T}$ of subtrees of $\bar{T}^{3}$ A subtree $T$ is defined by a subset of nodes $N \subseteq \bar{N}$ for which $(N, \lessdot)$ is also a tree (i.e., an arborescence in which a unique node has no predecessors). Two subtrees $T^{\prime}, T^{\prime \prime} \in \mathbf{T}$ are ordered, written

$$
T^{\prime} \preceq T^{\prime \prime}
$$

if the nodes of $T^{\prime}$ constitute a subset of the nodes of $T^{\prime \prime}$.

We require three properties:

1. All the terminal nodes in each tree $T \in \mathbf{T}$ are in $\bar{Z}$. That is, we don't create "new" terminal nodes.

2. For every tree $T \in \mathbf{T}$, every node $n \in T$, and every active player $i \in P(n)$ there exists a nonempty subset of actions $A_{i}^{T}(n) \subseteq A_{i}(n)$ such that $\psi_{n}$ maps the action profiles $A^{T}(n)=\prod_{i \in P(n)} A_{i}^{T}(n)$ bijectively onto $n$ 's successors in $T$.

were aware of it". It also avoids having to define a separate awareness correspondence that for each history specifies which histories the player is aware of.

${ }^{2}$ Alternatively, we could assign at every terminal node the same payoff to nature.

${ }^{3} \mathrm{~A}$ join semi-lattice is a partially ordered set in which each pair of elements has a join, i.e., a least upper bound. 
Figure 1: Property 1

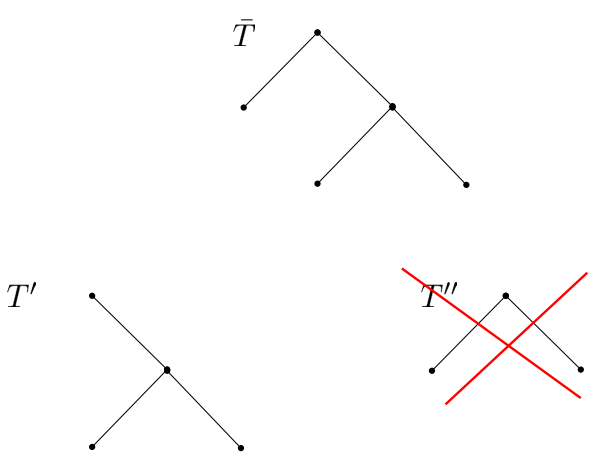

3. For any tree $T \in \mathbf{T}$, if for two decision nodes $n, n^{\prime} \in T$ with $i \in P(n) \cap P\left(n^{\prime}\right)$ it is the case that $A_{i}(n) \cap A_{i}\left(n^{\prime}\right) \neq \emptyset$, then $A_{i}(n)=A_{i}\left(n^{\prime}\right)$.

We illustrate Property 1 in Figure 1. Suppose that the tree the modeling all physical moves is given by $\bar{T}$. Then $T^{\prime}$ is a subtree satisfying Property 1 . In contrast, tree $T^{\prime \prime}$ does not satisfy Property 1 because it contains a new terminal node. After cutting branches from tree $\bar{T}$ to create tree $T^{\prime \prime}$, there is now a node in $T^{\prime \prime}$ that was not a terminal node in the original tree $\bar{T}$.

Within the family $\mathbf{T}$ of subtrees of $\bar{T}$, some nodes $n$ appear in several trees $T \in \mathbf{T}$. In what follows, we will need to designate explicitly appearances of such nodes $n$ in different trees as distinct objects. To this effect, in each tree $T \in \mathbf{T}$ label by $n_{T}$ the copy in $T$ of the node $n \in \bar{N}$ whenever the copy of $n$ is part of the tree $T$, with the requirement that if the profile of actions $a_{n} \in A(n)$ leads from $n$ to $n^{\prime}$, then $a_{n_{T}}$ leads also from the copy $n_{T}$ to the copy $n_{T}^{\prime}$. For any $T, T^{\prime}, T^{\prime \prime} \in \mathbf{T}$ with $T \preceq T^{\prime} \preceq T^{\prime \prime}$ such that $n \in T^{\prime \prime}, n_{T^{\prime}}$ is the copy of $n$ in the tree $T^{\prime}, n_{T}$ is the copy of $n$ in the tree $T$, and $\left(n_{T^{\prime}}\right)_{T}$ is the copy of $n_{T^{\prime}}$ in the tree $T$, we require that "nodes commute", $n_{T}=\left(n_{T^{\prime}}\right)_{T}$. For any $T \in \mathbf{T}$ and any $n \in T$, we let $n_{T}:=n$ (i.e., the copy of $n \in T$ in $T$ is $n$ itself).

Denote by $\mathbf{D}$ the union of all decision nodes in all trees $T \in \mathbf{T}$, by $\mathbf{Z}$ the union of terminal nodes in all trees $T \in \mathbf{T}$, and by $\mathbf{N}=\mathbf{D} \cup \mathbf{Z}$. Copies $n_{T}$ of a given node $n$ in different subtrees $T$ are now treated distinct from one another, so that $\mathbf{N}$ is a disjoint union of sets of nodes.

In what follows, when referring to a node in $\mathbf{N}$ we will typically avoid the subscript indicating the tree $T$ for which $n \in T$ when no confusion arises. For a node $n \in \mathbf{N}$ we denote by $T_{n}$ the tree containing $n .^{4}$

Denote by $N^{T}$ the set of nodes in the tree $T \in \mathbf{T}$. Similarly, denote by $D_{i}^{T}$ the set of decision nodes in which player $i$ is active in the tree $T \in \mathbf{T}$. Moreover, denote by $Z^{T}$ the set

\footnotetext{
${ }^{4}$ Bold capital letters refer to sets of elements across trees.
} 
of terminal nodes in the tree $T \in \mathbf{T}$. Finally, we let $\mathbf{D}_{i}$ the set of player $i$ 's decision nodes over all trees in $\mathbf{T}$. In the case of nature, $\mathbf{D}_{0}$ would be all nodes at which nature moves.

In extensive games with unawareness, information sets model both information and awareness. At decision node $n$ of player $i$ in the tree $T_{n} \in \mathbf{T}$, the player may conceive the feasible paths to be described by a different (i.e., less expressive) tree $T^{\prime} \in \mathbf{T}$. In such a case, her information set will be a subset of $T^{\prime}$ rather than of $T_{n}$ and $n$ will not be contained in the player's information set at $n$.

Formally, for each node $n \in \mathbf{N}$, define for each active player $i \in P(n) \backslash\{0\}$ a nonempty information set $h_{i}(n)$ with the following properties: ${ }^{5}$

U0 Confined awareness: If $n \in T$ and $i \in P(n)$, then $h_{i}(n) \subseteq T^{\prime}$ with $T^{\prime} \preceq T$.

U1 Generalized reflexivity: If $T^{\prime} \preceq T, n \in T, h_{i}(n) \subseteq T^{\prime}$ and $T^{\prime}$ contains a copy $n_{T^{\prime}}$ of $n$, then $n_{T^{\prime}} \in h_{i}(n)$.

I2 Introspection: If $n^{\prime} \in h_{i}(n)$, then $h_{i}\left(n^{\prime}\right)=h_{i}(n)$.

I3 No divining of currently unimaginable paths, no expectation to forget currently conceivable paths: If $n^{\prime} \in h_{i}(n) \subseteq T^{\prime}$ (where $T^{\prime} \in \mathbf{T}$ is a tree) and there is a path $n^{\prime}, \ldots, n^{\prime \prime} \in T^{\prime}$ such that $i \in P\left(n^{\prime}\right) \cap P\left(n^{\prime \prime}\right)$, then $h_{i}\left(n^{\prime \prime}\right) \subseteq T^{\prime}$.

I4 No imaginary actions: If $n^{\prime} \in h_{i}(n)$, then $A_{i}\left(n^{\prime}\right) \subseteq A_{i}(n)$.

I5 Distinct action names in disjoint information sets: For a subtree $T \in \mathbf{T}$, if there a decision nodes $n, n^{\prime} \in T \cap \mathbf{D}$ with $A_{i}(n)=A_{i}\left(n^{\prime}\right)$, then $h_{i}\left(n^{\prime}\right)=h_{i}(n)$.

I6 Perfect recall: Suppose that player $i$ is active in two distinct nodes $n_{1}$ and $n_{k}$, and there is a path $n_{1}, n_{2}, \ldots, n_{k}$ such that at $n_{1}$ player $i$ takes the action $a_{i}$. If $n^{\prime} \in h_{i}\left(n_{k}\right), n^{\prime} \neq n_{k}$, then there exists a node $n_{1}^{\prime} \neq n^{\prime}$ and a path $n_{1}^{\prime}, n_{2}^{\prime}, \ldots, n_{\ell}^{\prime}=n^{\prime}$ such that $h_{i}\left(n_{1}^{\prime}\right)=h_{i}\left(n_{1}\right)$ and at $n_{1}^{\prime}$ player $i$ takes the action $a_{i}$.

Properties (I2), (I4), and (I5) are standard for extensive games, and properties (U0), (U1), and (I6) generalize standard properties of extensive games to our generalized setting. At each information set of a player, property (I3) confines the player's anticipation of her future view of the game to the view she currently holds (even if, as a matter of fact, this view is about to be shattered as the game evolves).

Central to our extension of Kuhn's Theorem is the assumption of perfect recall (I6). This property is illustrated with an example and a counterexample in Figure 2. It is known that in standard extensive games without unawareness, perfect recall is necessary for the "playability" of strategies and the existence of Nash equilibrium in behavior strategies; see for instance

\footnotetext{
${ }^{5}$ We keep the numbering consistent with Heifetz, Meier, and Schipper (2013).
} 
Figure 2: Perfect Recall
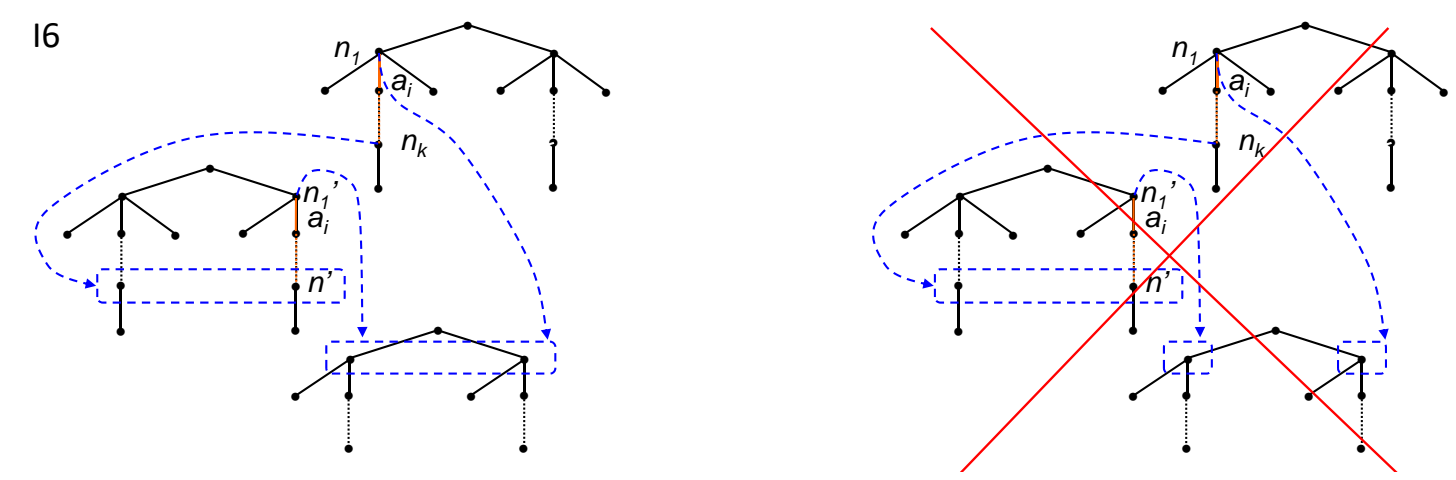

Wichardt (2008). Since standard extensive games are special cases of extensive games with unawareness, such arguments apply also to extensive games with unawareness.

Figure 3 illustrates with an example and a counterexample each both U0 and U1.

Figure 3: Properties U0 and U1
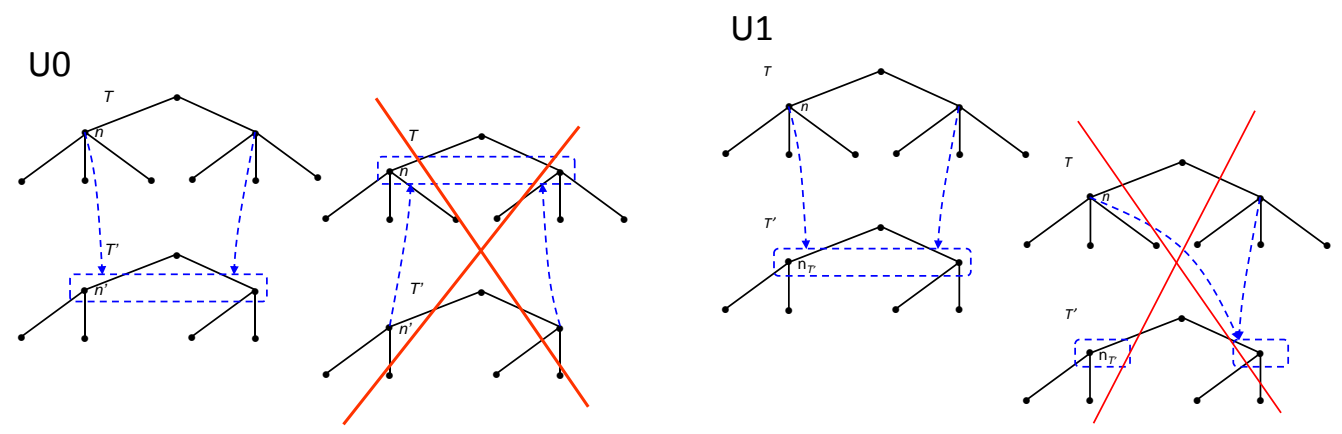

We denote by $H_{i}$ the set of player $i$ 's information sets in all trees. For an information set $h_{i} \in H_{i}$, we denote by $T_{h_{i}}$ the tree containing $h_{i}$. For two information sets $h_{i}, h_{i}^{\prime}$ in a given tree $T$, we say that $h_{i}$ precedes $h_{i}^{\prime}$ (or that $h_{i}^{\prime}$ succeeds $h_{i}$ ) if for every $n^{\prime} \in h_{i}^{\prime}$ there is a path $n, \ldots, n^{\prime}$ in $T$ such that $n \in h_{i}$. We denote it by $h_{i} \rightsquigarrow h_{i}^{\prime}$.

The following property is implied by I2 and I4 (see Heifetz, Meier, and Schipper, 2013, Remark 1): If $n^{\prime}, n^{\prime \prime} \in h_{i}$ where $h_{i}=h_{i}(n)$ is an information set for some $n \in \mathbf{D}_{i}$, then $A_{i}\left(n^{\prime}\right)=A_{i}\left(n^{\prime \prime}\right)$. Hence, if $n \in h_{i}$ we write also $A_{i}\left(h_{i}\right)$ for $A_{i}(n)$.

Properties U0, U1, I2, and I6 imply no absent-mindedness. This follows directly from Heifetz, Meier, and Schipper (2013, Remark 2).

No Absent-mindedness: No information set $h_{i}$ contains two distinct nodes $n, n^{\prime}$ on the 
same path in some tree.

The Perfect recall property I6 and no absent-mindedness guarantee that with the precedence relation $\rightsquigarrow$ player $i$ 's information sets $H_{i}$ form an arborescence: For every information set $h_{i}^{\prime} \in H_{i}$, the information sets preceding it $\left\{h_{i} \in H_{i}: h_{i} \rightsquigarrow h_{i}^{\prime}\right\}$ are totally ordered by $\rightsquigarrow$.

Perfect recall can be interpreted as players do not forget their experience throughout the game. This can be made explicit. For any player $i \in I$ and decision node of that player $n \in \mathbf{D}_{i}$, let $E_{i}(n)$ denote the record of player $i$ 's experience along the path to $n$ (not including $h_{i}(n)$ ). I.e., $E_{i}(n)$ is the sequence of pairs $\left(h_{i}, a_{i}\right)$ of player $i$ 's information sets and the action taken at these information sets in order of how they are encountered along the path to $n$. Perfect recall is now characterized as follows:

Remark 1 An extensive game with unawareness satisfies perfect recall (I6) if and only if for any player $i \in I, n \in \mathbf{D}_{i}, n^{\prime} \in h_{i}(n)$ implies $E_{i}\left(n^{\prime}\right)=E_{i}(n)$.

Proof. " $\Rightarrow "$ : Consider the non-trivial case $n^{\prime} \neq n$. Suppose by contradiction that $E_{i}\left(n^{\prime}\right) \neq$ $E_{i}(n)$. Then there exists a path $n_{1}, n_{2}, \ldots, n_{k}$ with $n=n_{k}$ for which there is no path $n_{1}^{\prime}, n_{2}^{\prime}, \ldots, n_{\ell}^{\prime}$ with $n^{\prime}=n_{\ell}^{\prime}$ such that $h_{i}\left(n_{1}\right)=h_{i}\left(n_{1}^{\prime}\right)$ and the action taken at $n_{1}^{\prime}$ along the path $n_{1}^{\prime}, n_{2}^{\prime}$ is the action taken at $n_{1}$ along the path $n_{1}, n_{2}$. But this contradicts I6.

" $\Leftarrow$ ": Suppose not. Then for any two nodes $n_{1}$ and $n_{k}$ with $n_{1} \neq n_{k}$ with the path $n_{1}, n_{2}, \ldots, n_{k}$ such that at $n_{1}$ player $i$ takes action $a_{i}$ along the path, $n^{\prime} \in h_{i}\left(n_{k}\right), n^{\prime} \neq n_{k}$, there is no node $n_{1}^{\prime} \neq n^{\prime}$ and a path $n_{1}^{\prime}, n_{2}^{\prime}, \ldots, n_{\ell}^{\prime}=n^{\prime}$ such that $h_{i}\left(n_{1}^{\prime}\right)=h_{i}\left(n_{1}\right)$ and player $i$ takes action $a_{i}$ at $n_{1}^{\prime}$ along the path. But this just means that player's records of experience are different in nodes $n$ and $n^{\prime}$, i.e., $E_{i}\left(n^{\prime}\right) \neq E_{i}(n)$, a contradiction.

Confined awareness (U0) and Perfect recall (I6) imply that a player cannot become unaware during the play (see Heifetz, Meier, and Schipper, 2013, Remark 6).

DA Awareness may only increase along a path: If there is a path $n, \ldots, n^{\prime}$ in some subtree $T^{\prime \prime}$ such that player $i$ is active in $n$ and $n^{\prime}$, and $h_{i}(n) \subseteq T$ while $h_{i}\left(n^{\prime}\right) \subseteq T^{\prime}$, then $T^{\prime} \succeq T$.

To model unawareness proper, we impose as in Heifetz, Meier, and Schipper (2013) additional properties. They parallel properties of static unawareness structures in Heifetz, Meier, and Schipper (2006):

U4 Subtrees preserve ignorance: If $T \preceq T^{\prime} \preceq T^{\prime \prime}, n \in T^{\prime \prime}, h_{i}(n) \subseteq T$ and $T^{\prime}$ contains the copy $n_{T^{\prime}}$ of $n$, then $h_{i}\left(n_{T^{\prime}}\right)=h_{i}(n)$.

U5 Subtrees preserve knowledge: If $T \preceq T^{\prime} \preceq T^{\prime \prime}, n \in T^{\prime \prime}, h_{i}(n) \subseteq T^{\prime}$ and $T$ contains the copy $n_{T}$ of $n$, then $h_{i}\left(n_{T}\right)$ consists of the copies that exist in $T$ of the nodes of $h_{i}(n)$. 
It is known that U5 implies U3, see Heifetz, Meier, and Schipper (2013, Remark 3):

U3 Subtrees preserve awareness: If $n \in T^{\prime}, n \in h_{i}(n), T \preceq T^{\prime}$, and $T$ contains a copy $n_{T}$ of $n$, then $n_{T} \in h_{i}\left(n_{T}\right)$.

Properties U3 to U5 are illustrated in Figure 4 with an example and counterexample each.

Figure 4: Properties U3 to U5
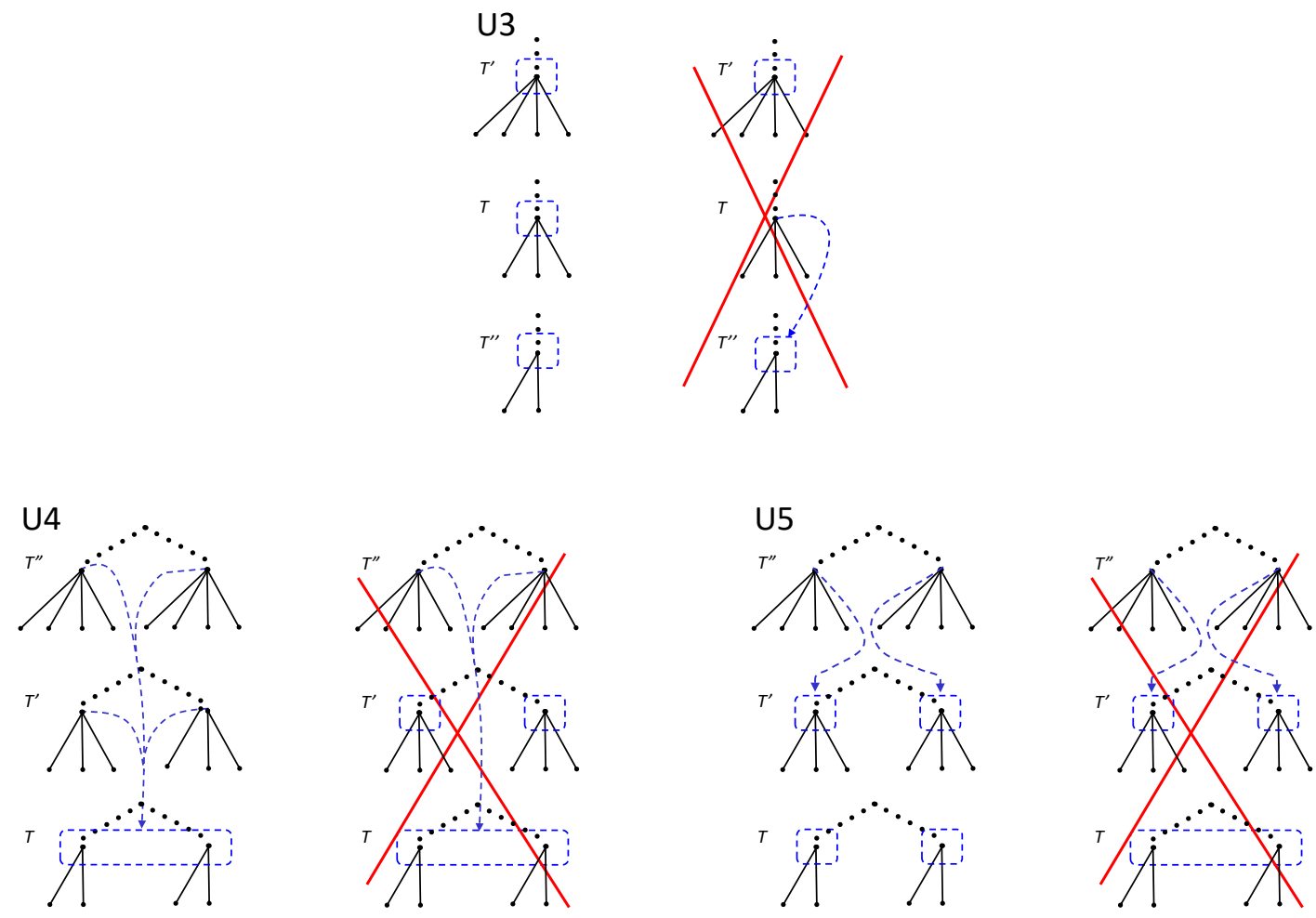

For trees $T, T^{\prime} \in \mathbf{T}$ we denote by $T \longmapsto T^{\prime}$ whenever for some node $n \in T$ and some player $i \in P(n)$ it is the case that $h_{i}(n) \subseteq T^{\prime}$. Denote by $\hookrightarrow$ the transitive closure of $\hookrightarrow$. That is, $T \hookrightarrow$ $T^{\prime \prime}$ if and only if there is a sequence of trees $T, T^{\prime}, \ldots, T^{\prime \prime} \in \mathbf{T}$ satisfying $T \longmapsto T^{\prime} \longmapsto \cdots \longmapsto T^{\prime \prime}$.

An extensive game with unawareness $\Gamma$ consists of a join-semilattice $\mathbf{T}$ of subtrees of a tree $\bar{T}$ satisfying properties 1-3 above, along with information sets $h_{i}(n)$ for every $n \in T$ with $T \in \mathbf{T}$ and $i \in P(n)$, and payoffs satisfying properties $\mathrm{U} 0, \mathrm{U} 1, \mathrm{U} 4, \mathrm{U} 5$, and I2-I7 above.

For any extensive game with unawareness $\Gamma$ with set of trees $\mathbf{T}$, for any tree $T \in \mathbf{T}$, the $T$ partial game is the join-semisublattice of trees including $T$ and also all trees $T^{\prime}$ in $\Gamma$ satisfying $T \hookrightarrow T^{\prime}$, with information sets as defined in $\Gamma$. A $T$-partial game is a extensive game with unawareness, i.e., it satisfies all properties 1-3, U0, U1, U4, U5, and I2-I7 above.

We denote by $H_{i}^{T}$ the set of player $i$ 's information sets in the $T$-partial game, $T \in \mathbf{T}$. This 
set contains not only $i$ 's information sets in the tree $T$ but also in all trees $T^{\prime} \in \mathbf{T}$ with $T \hookrightarrow T^{\prime}$.

\section{Strategies}

For any collection of sets $\left(X_{i}\right)_{i \in I^{0}}$ we denote by

$$
X:=\prod_{i \in I^{0}} X_{i}, \quad X_{-i}:=\prod_{j \in I^{0} \backslash\{i\}} X_{j}
$$

with typical elements $x$ and $x_{-i}$ respectively. For any collection of sets $\left(X_{i}\right)_{i \in I^{0}}$ and any tree

$T \in \mathbf{T}$, we denote by $X_{i}^{T}$ the set of objects in $X_{i}$ restricted to the tree $T$ and analogously for $X^{T}$ and $X_{-i}^{T}$, where "restricted to the tree $T$ " will become clear from the definitions below.

A pure strategy for player iinI,

$$
s_{i} \in S_{i}:=\prod_{h_{i} \in H_{i}} A\left(h_{i}\right)
$$

specifies an action of player $i$ at each of her information sets $h_{i} \in H_{i}$. We let

$$
s_{0} \in S_{0}:=\prod_{n \in \mathbf{D}_{0}} A_{0}(n)
$$

denote the "strategy" of nature, with $\mathbf{D}_{0}$ denoting the "decision" nodes of nature.

With the strategy $s_{i}$, at node $n \in D_{i}^{T_{n}}$ define player $i$ 's action at $n$ to be $s_{i}\left(h_{i}(n)\right)$, for $i \in I$. Thus, by U1 and I4 the strategy $s_{i}$ specifies what player $i \in I$ does at each of her active nodes $n \in D_{i}^{T_{n}}$, both in the case that $n \in h_{i}(n)$ and in the case that $h_{i}(n)$ is a subset of nodes of a tree which is distinct from the tree $T_{n}$ to which $n$ belongs. In the first case, when $n \in h_{i}(n)$, we can interpret $s_{i}\left(h_{i}(n)\right)$ as the action chosen by player $i$ in node $n$. In the second case, when $n \notin h_{i}(n), s_{i}\left(h_{i}(n)\right)$ cannot be interpreted as the action chosen "consciously" by player $i$ in $n$ since she is not even aware of $T_{n}$. Instead, her state of mind at $n$ is given by her information set $h_{i}(n)$ in a tree lower than $T_{n}$ (denoted by $\left.T_{h_{i}(n)}\right)$. Thus, $s_{i}\left(h_{i}(n)\right)$ is the physical move of player $i$ in $n$ in tree $T_{n}$ induced by her "consciously" chosen action at her information set $h_{i}(n)$ in tree $T_{h_{i}(n)}$ (with $T_{n} \succ T_{h_{i}(n)}$ ). As an example, consider the game in Figure 5 . The information set at node $n$ in tree $T_{n}$ lies in the lower tree $T_{h(n)}$ that misses action "middle". This is indicated by the blue arrow and disk. When the player chooses "left" in $T_{h(n)}$ (as indicated by the red solid line beside the left edge), it induces also an action "left" at node $n$ in tree $T_{n}$ (as indicated by the red dashed line beside the left edge).

In an extensive game with unawareness $\Gamma$ the tree $\bar{T} \in \mathbf{T}$ represents the physical paths in the game; every tree in $\mathbf{T}$ that contains an information set represents the subjective view of the feasible paths in the mind of a player, or the view of the feasible paths that a player believes that another player may have in mind, etc. Moreover, as the actual play in $\bar{T}$ unfolds, a player 
Figure 5: Action induced by a strategy

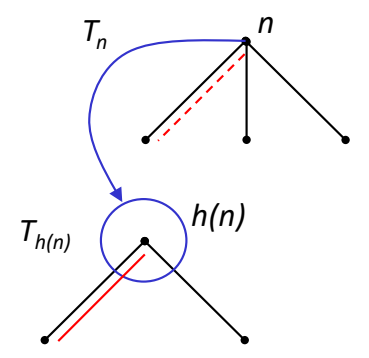

may become aware of paths of which she was unaware earlier, and the way she views the game may be altered. Thus, in an extensive game with unawareness, a strategy cannot be conceived as an ex ante plan of action. Formally, a strategy of player $i$ is a list of answers to the questions "what would player $i \in I$ do if $h_{i}$ were the set of nodes she considered as possible?", for $h_{i} \in H_{i}$ (and analogous for nature). A strategy of a player becomes meaningful as an object of beliefs of other players. How "much" of a player's strategy other players can conceive depend on their awareness given by the tree in which their information set is located. This leads to the notion of $T$-partial strategy. For a strategy $s_{i} \in S_{i}$ and a tree $T \in \mathbf{T}$, we denote by $s_{i}^{T}$ the strategy in the $T$-partial game induced by $s_{i}$ (i.e., $s_{i}^{T}\left(h_{i}\right)=s_{i}\left(h_{i}\right)$ for every information set $h_{i} \in H_{i}^{T}$ of player $i$ in the $T$-partial game). (Recall that $H_{i}^{T}$ not only contains information sets in the tree $T$ but also in trees $T^{\prime} \in \mathbf{T}$ with $T^{\prime} \preceq T$.)

A mixed strategy of player $i, \sigma_{i} \in \Delta\left(S_{i}\right)$, specifies a probability distribution over player $i$ 's set of pure strategies. With this notation, we let $\sigma_{0}$ the probability distribution over "strategies" of nature. As mentioned already in the introduction, we don't consider mixed strategies necessarily as an object of choice of players but rather a conjecture over how a player would play.

A behavior strategy for player $i \in I$,

$$
\beta_{i} \in B_{i}:=\prod_{h_{i} \in H_{i}} \Delta\left(A_{i}\left(h_{i}\right)\right)
$$

is a collection of independent probability distributions, one for each of player $i$ 's information sets $h_{i} \in H_{i}$, where $\beta_{i}\left(h_{i}\right)$ specifies a mixed action in $\Delta\left(A_{h_{i}}\right)$. With the behavior strategy $\beta_{i}$, at node $n \in D_{i}^{T_{n}}$ define player $i$ 's mixed action at $n$ to be $\beta_{i}\left(h_{i}(n)\right)$. Thus, the behavior strategy $\beta_{i}$ specifies the mixed action of player $i \in I$ at each of her active decision nodes $n \in D_{i}^{T_{n}}$, both in the case that $n \in h_{i}(n)$ and in the case that $h_{i}(n)$ is a subset of nodes of a tree which is distinct from the tree $T_{n}$ to which $n$ belongs. It may be the case that $A_{i}(n) \supset A_{i}\left(h_{i}(n)\right)$. Yet, we have automatically that $\beta_{i}$ does not assign probabilities to actions in $A_{n} \backslash A_{h_{i}(n)}$. (I.e., at the decision node $n$ of the richer tree $T_{n}$ player $i$ may have more actions than she is aware of at $h_{i}(n)$. In such a case, she is unable to use actions that she is unaware of.) With respect to nature, we let $\beta_{0} \in B_{0}=\prod_{n \in D_{0}} \Delta\left(A_{0}(n)\right)$. 
We say that a strategy profile $s=\left(s_{j}\right)_{j \in I} \in S$ reaches a node $n \in T$ if the players' actions and nature's moves $\left(s_{j}^{T}\left(h_{j}\left(n^{\prime}\right)\right)\right)_{j \in P\left(n^{\prime}\right)}$ in nodes $n^{\prime} \in T$ lead to $n$. Notice that by property (I4) ("no imaginary actions"), $s_{j}^{T}\left(h_{j}\left(n^{\prime}\right)\right)_{j \in I}$ is indeed well defined: even if $h_{j}\left(n^{\prime}\right) \nsubseteq T$ for some $n^{\prime} \in T,\left(s_{j}^{T}\left(h_{j}\left(n^{\prime}\right)\right)\right)_{j \in P\left(n^{\prime}\right)}$ is a profile of actions which is actually available in $T$ to the active players $j \in P\left(n^{\prime}\right)$ and possibly nature at $n^{\prime}$. We say that a strategy profile $s \in S$ reaches the information set $h_{i} \in H_{i}$ if $s$ reaches some node $n \in h_{i}$. We say that the strategy $s_{i} \in S_{i}$ reaches the information set $h_{i}$ if there is a strategy profile $s_{-i} \in S_{-i}$ of the other players (and possibly nature) such that the strategy profile $\left(s_{i}, s_{-i}\right)$ reaches $h_{i}$. Analogously, we say that the strategy profile $s_{-i} \in S_{-i}$ reaches the information set $h_{i}$ if there exists a strategy $s_{i} \in S_{i}$ such that the strategy profile $\left(s_{i}, s_{-i}\right)$ reaches $h_{i}$. For each player $i \in I$, denote by $H_{i}(s)$ the set of information sets of $i$ that are reached by the strategy profile $s$. This set may contain information sets in more than one tree.

We extend the definitions of information set reached to mixed and behavior strategies in the obvious way by considering nodes/information sets reached with strict positive probability.

\section{Kuhn's Theorem}

In extensive games without unawareness but with perfect recall, Kuhn's Theorem asserts that for every mixed strategy profile there is an equivalent behavior strategy profile. Kuhn's Theorem can be extended to extensive games with unawareness using a notion of equivalence based on the notion of reaching nodes. For any node $n$, any player $i \in I^{0}$, and any opponents' profile of strategies $s_{-i}$ (including nature if any), let $\rho\left(n \mid \beta_{i}, s_{-i}\right)$ and $\rho\left(n \mid \sigma_{i}, s_{-i}\right)$ denote the probability that $\left(\beta_{i}, s_{-i}\right)$ and $\left(\sigma_{i}, s_{-i}\right)$ reach node $n$, respectively. For any player $i \in I^{0}$, a mixed strategy $\sigma_{i}$ and a behavior strategy $\beta_{i}$ are equivalent if for every profile of opponents' strategies $s_{-i} \in S_{-i}$ and every node $n \in \mathbf{N}$ of the extensive game with unawareness $\rho\left(n \mid \sigma_{i}, s_{-i}\right)=\rho\left(n \mid \beta_{i}, s_{-i}\right)$.

Let $S_{i}(n)$ be the set of all strategies of player $i$ that reach $n$. That is, if $n \in T$ then $s_{i} \in S_{i}(n)$ if and only there exist $s_{-i} \in S_{-i}$ such that the profile $\left(s_{j}^{T}\left(h_{j}\left(n^{\prime}\right)\right)\right)_{j \in P\left(n^{\prime}\right)}$ in $n^{\prime} \in T$ lead to $n$.

In the following, we adapt for better comparison a textbook proof of Kuhn's Theorem (e.g., Maschler, Solan, and Zamir, 2013, Chapter 6) to the more complicated set up of extensive games with unawareness. It allows us to show which modifications are necessary as compared to standard games, and emphasizes that the basic idea of proof remains the same. We start with a lemma that is crucial for the proof of the main theorem.

Lemma 1 Consider an extensive game with unawareness $\Gamma$. If $\Gamma$ satisfies perfect recall (i.e., I6), then for any player $i \in I, n \in N$ with $h_{i}(n) \in H_{i}$, and $n^{\prime} \in h_{i}(n), S_{i}(n)=S_{i}\left(n^{\prime}\right)$.

Note the difference to standard games. First, extensive games with unawareness involve 
forests of trees rather than just trees. Second, the perfect recall property applies now to information sets across trees. Third, the sets $S_{i}(n)$ are different from corresponding sets in standard games because $n$ may be in a tree different from the tree "housing" the information set $h_{i}(n)$ of player $i$ at $n$. This is significant because strategies are defined for each information set (rather than decision node) of player $i$.

Proof of Lemma 1. Since $\Gamma$ satisfies perfect recall, we have by Remark 1 that $n^{\prime} \in h_{i}(n)$ implies $E_{i}\left(n^{\prime}\right)=E_{i}(n)$. Thus, the same information sets of player $i$ are reached along the path to $n$ that are reached along the path to $n^{\prime}$ (even though the information sets might appear in a subtree lower than the one containing the path to $n$ ). Moreover, at each of those information sets the same action is required to move along the path to $n$ as to move along to the path to $n^{\prime}$. Hence, any strategy reaching $n$ also reaches $n^{\prime}$ and vice versa.

We are now ready to state an extension of Kuhn's Theorem to extensive games with unawareness.

Theorem 1 In every extensive game with unawareness, if player $i$ has perfect recall, then for every mixed strategy of player $i$ there exists an equivalent behavior strategy.

Proof. The theorem is proved in three steps. The first step defines the candidate of the behavior strategy. The second step shows that it is well-defined. The third step shows it to be equivalent to the mixed strategy.

Let $\sigma_{i}$ be a mixed strategy of player $i$.

First, we define a candidate for the equivalent behavior strategy. Let $n \in \mathbf{D}_{i}$. Since $n \in \mathbf{D}_{i}$ we have $A_{i}(n) \neq \emptyset$. For any action $a_{i} \in A_{i}(n)$ of player $i$ at $n$, define $S_{i}\left(n, a_{i}\right):=\left\{s_{i} \in S_{i}(n)\right.$ : $\left.s_{i}\left(h_{i}(n)\right)=a_{i}\right\}$. That is, any strategy in $S_{i}\left(n, a_{i}\right)$ reaches $n$ and also prescribes action $a_{i}$ at information set $h_{i}(n)$. This definition makes sense: First, recall that strategies ascribe actions to information sets (rather than nodes). Moreover, by definition of $\Gamma$ there is an information set of player $i$ at $n$ that we denote by $h_{i}(n)$. There are two cases: First, $n \in h_{i}(n)$. In this case, for any $n^{\prime} \in h_{i}(n)$ we have $A_{i}(n)=A_{i}\left(n^{\prime}\right)$ (Heifetz, Meier, and Schipper, 2013, Remark 1). Thus, we write $A_{i}\left(h_{i}(n)\right)$ for actions available at any node in $h_{i}(n)$. Second, $n \notin h_{i}(n)$. (That's the case when $n$ is in a tree more expressive than $h_{i}(n)$.) By I4 (No imaginary actions), for any $n^{\prime} \in h_{i}(n)$ we have $A_{i}\left(n^{\prime}\right) \subseteq A_{i}(n)$. Note also that $A_{i}\left(n^{\prime}\right) \neq \emptyset$ since $n^{\prime} \in \mathbf{D}_{i}$. If $a_{i} \in A_{i}(n) \backslash A_{i}\left(n^{\prime}\right)$, then $S_{i}\left(n, a_{i}\right)=\emptyset$ since no strategy of player $i$ can ascribe an action to $n$ that is not available at $h_{i}(n)$. Hence, in the following we consider sets $S_{i}\left(n, a_{i}\right)$ for $a_{i} \in A_{i}\left(h_{i}(n)\right)$.

If player $i$ 's mixed strategy assigns strict positive probability to strategies reaching $n$, i.e., 
if $\sum_{s_{i} \in S_{i}(n)} \sigma_{i}\left(s_{i}\right)>0$, then define for each action $a_{i} \in A_{i}\left(h_{i}(n)\right)$,

$$
\beta_{i}\left(h_{i}(n)\right)\left(a_{i}\right):=\frac{\sum_{s_{i} \in S_{i}\left(n, a_{i}\right)} \sigma_{i}\left(s_{i}\right)}{\sum_{s_{i} \in S_{i}(n)} \sigma_{i}\left(s_{i}\right)} .
$$

Otherwise, if $\sum_{s_{i} \in S_{i}(n)} \sigma_{i}\left(s_{i}\right)=0$, define $\beta_{i}\left(h_{i}(n)\right)$ in an arbitrary way provided that it constitutes a probability measure over actions available at $h_{i}(n)$. E.g., for all $a_{i} \in A_{i}\left(h_{i}(n)\right)$,

$$
\beta_{i}\left(h_{i}(n)\right)\left(a_{i}\right):=\frac{1}{\left|A_{i}\left(h_{i}(n)\right)\right|} .
$$

Second, we want to show that $\beta_{i}$ is well-defined. I.e., for each of player $i$ 's information sets $h_{i} \in H_{i}, \beta_{i}\left(h_{i}\right)$ is a probability measure on $A_{i}\left(h_{i}\right)$. Moreover, $\beta_{i}$ is independent of player $i$ 's decision nodes. For the latter, it suffices to demonstrate it for the case $\sum_{s_{i} \in S_{i}(n)} \sigma_{i}\left(s_{i}\right)>0$. Since $\Gamma$ has perfect recall, i.e., $\Gamma$ satisfies I6, we have by Lemma 1 that for any $n^{\prime} \in h_{i}(n)$, $S_{i}\left(n^{\prime}\right)=S_{i}(n)$. Again, since $n^{\prime} \in h_{i}(n), S_{i}\left(n, a_{i}\right)=S_{i}\left(n^{\prime}, a_{i}\right)$ for all $a_{i} \in A_{i}\left(h_{i}\right)$. Observe that both the numerator and the denominator of the left-hand side of Equation (1) are independent of nodes in $h_{i}(n)$.

To show that for all $n \in \mathbf{D}_{i}, \beta_{i}\left(h_{i}(n)\right)$ is a probability measure over $A_{i}\left(h_{i}(n)\right)$, note first that, in the case of $\sum_{s_{i} \in S_{i}(n)} \sigma_{i}\left(s_{i}\right)=0$, this follows directly from Equation (2).

If $\sum_{s_{i} \in S_{i}(n)} \sigma_{i}\left(s_{i}\right)>0$, then Equation (1) defines a probability distribution over $A_{i}\left(h_{i}\right)$. To see this note that since $\sigma_{i}\left(s_{i}\right) \geq 0$ for all $s_{i} \in S_{i}$, both the numerator and the denominator are nonnegative and hence $\beta_{i}\left(h_{i}\right)\left(a_{i}\right) \geq 0$ for all $a_{i} \in A_{i}\left(h_{i}\right)$. For any $n \in \mathbf{D}_{i}$ and $a_{i}, a_{i}^{\prime} \in$ $A_{i}\left(h_{i}(n)\right)$ with $a_{i} \neq a_{i}^{\prime}, S_{i}\left(n, a_{i}\right) \cap S_{i}\left(n, a_{i}^{\prime}\right)=\emptyset$. Moreover, $\bigcup_{a_{i} \in A_{i}\left(h_{i}(n)\right)} S_{i}\left(n, a_{i}\right)=S_{i}(n)$. Thus, $\sum_{a_{i} \in A_{i}\left(h_{i}(n)\right)} \sum_{s_{i} \in S_{i}\left(n, a_{i}\right)} \sigma_{i}\left(s_{i}\right)=\sum_{s_{i} \in S_{i}(n)} \sigma_{i}\left(s_{i}\right)$. If follows that $\sum_{a_{i} \in A_{i}\left(h_{i}\right)} \beta_{i}\left(h_{i}\right)\left(a_{i}\right)=1$.

The third and last step is to show that the behavior strategy $\beta_{i}$ is equivalent to the mixed strategy $\sigma_{i}$. Fix a node $n \in \mathbf{D}_{i} \cup \mathbf{Z}$ and let $n_{i}^{1}, n_{i}^{2}, \ldots, n_{i}^{L}$ be a sequence of decision nodes of player $i$ along the path from the root to $n$, not including $n$. By definition of $\Gamma$, there exist an information set of player $i$ for each of the decision nodes $n_{i}^{1}, n_{i}^{2}, \ldots, n_{i}^{L}$. If $L=0$, then player $i$ has no information sets on the path from the root to $n$ (not including $n$ ). In such a case, $S_{i}(n)=S_{i}$ and we naturally define $\rho\left(n \mid \beta_{i}, s_{-i}\right)=1$. Also, in this case $\rho\left(n \mid \sigma_{i}, s_{-i}\right)=$ $\sum_{s_{i} \in S_{i}(n)} \sigma_{i}\left(s_{i}\right)=\Sigma_{s_{i} \in S_{i}} \sigma_{i}\left(s_{i}\right)=1$. Hence, $\beta_{i}$ and $\sigma_{i}$ are equivalent in this case.

Suppose now the case $L>0$. Let $a_{i}^{n_{i}^{\ell}} \in A_{i}\left(h_{i}\left(n_{i}^{\ell}\right)\right)$ denote the action of player $i$ at node $n_{i}^{\ell}$, $\ell=1, \ldots, L$, that leads to $n_{i}^{\ell+1}$ in the case $\ell=1, \ldots, L-1$ and to $n$ in the case $\ell=L$.

We have

$$
\rho\left(n \mid \beta_{i}, s_{-i}\right)=\prod_{\ell=1}^{L} \beta_{i}\left(h_{i}\left(n_{i}^{\ell}\right)\right)\left(a_{i}^{n_{i}^{\ell}}\right) .
$$

Assume that $\sigma_{i}$ reaches $n$. By definition of $\beta_{i}$,

$$
\rho\left(n \mid \beta_{i}, s_{-i}\right)=\prod_{\ell=1}^{L} \frac{\sum_{s_{i} \in S_{i}\left(n_{i}^{\ell}, n_{i}^{n_{i}^{\ell}}\right)} \sigma_{i}\left(s_{i}\right)}{\sum_{s_{i} \in S_{i}\left(n_{i}^{\ell}\right)} \sigma_{i}\left(s_{i}\right)},
$$


which is well-defined since $\sigma_{i}$ is assumed to reach $n$ and therefore also to reach $n_{i}^{1}, \ldots, n_{i}^{L}$.

Note that $S_{i}\left(n_{i}^{\ell+1}\right)=S_{i}\left(n_{i}^{\ell}, a_{i}^{\ell}\right)$. Thus

$$
\sum_{s_{i} \in S_{i}\left(n_{i}^{\ell}, a_{i}^{\ell}\right)} \sigma_{i}\left(s_{i}\right)=\sum_{s_{i} \in S_{i}\left(n_{i}^{\ell+1}\right)} \sigma_{i}\left(s_{i}\right)
$$

and

$$
\rho\left(n \mid \beta_{i}, s_{-i}\right)=\prod_{\ell=1}^{L} \frac{\sum_{s_{i} \in S_{i}\left(n_{i}^{\ell+1}\right)} \sigma_{i}\left(s_{i}\right)}{\sum_{s_{i} \in S_{i}\left(n_{i}^{\ell}\right)} \sigma_{i}\left(s_{i}\right)},
$$

(where we take $n_{L+1}=n$ ) is the telescopic product for which the numerator of the $\ell$-th term of the product equals to the denominator of the $\ell+1$-th term of the product. Adjacent product terms cancel each other out. Thus,

$$
\rho\left(n \mid \beta_{i}, s_{-i}\right)=\frac{\sum_{s_{i} \in S_{i}(n)} \sigma_{i}\left(s_{i}\right)}{\sum_{s_{i} \in S_{i}\left(n_{i}^{1}\right)} \sigma_{i}\left(s_{i}\right)} .
$$

Since $n_{i}^{1}$ is in player $i$ 's first information set on the path towards $n$, we have $S_{i}\left(n_{1}\right)=S_{i}$. Hence,

$$
\rho\left(n \mid \beta_{i}, s_{-i}\right)=\frac{\sum_{s_{i} \in S_{i}(n)} \sigma_{i}\left(s_{i}\right)}{\sum_{s_{i} \in S_{i}} \sigma_{i}\left(s_{i}\right)} .
$$

Since trivially any strategy of player $i$ reaches her first information set, we have $\Sigma_{s_{i} \in S_{i}} \sigma_{i}\left(s_{i}\right)=1$. Thus,

$$
\rho\left(n \mid \beta_{i}, s_{-i}\right)=\frac{\sum_{s_{i} \in S_{i}(n)} \sigma_{i}\left(s_{i}\right)}{1}=\rho_{i}\left(n \mid \sigma_{i}, s_{-i}\right)
$$

This completes the proof of the theorem.

\subsection{Equivalence in Realization of Nodes}

In extensive games with unawareness there are two distinct notions of a strategy profile being consistent with a node. The first notion we introduced already at the end of Section 3 and called it a "strategy profile reaching a node". While a player may expect a strategy profile to reach a node, it can be the case in games with unawareness that a different node actually occurs. This is because the player is unaware of actions that a player with more awareness may take (since each player just considers the partial strategies consistent with her awareness level). This begs the question whether strategies that are equivalent with respect to nodes reached are also equivalent with respect to nodes that actually occur. Note that both notions of a node being consistent with a strategy are relevant. The notion of a strategy reaching a node is relevant for extensive rationalizability (extended to extensive games with unawareness by Heifetz, Meier, and Schipper, 2013) whereas the notion of a node occurring with a strategy 
is crucial for self-confirming equilibrium (as extended to extensive games with unawareness by Schipper, 2017).

We say that node $n \in \bar{T}$ in the upmost tree $\bar{T}$ occurs with strategy profile $s=\left(s_{j}\right)_{j \in I} \in S$ if the players' actions and nature's moves $\left(s_{j}\left(h_{j}\left(n^{\prime}\right)\right)\right)_{j \in P\left(n^{\prime}\right)}$ in nodes $n^{\prime} \in \bar{T}$ lead to $n \in \bar{T}$. We extend the notion to any node in any tree by saying that node $n \in T$ occurs with strategy profile $s=\left(s_{j}\right)_{j \in I} \in S$ if there is $n^{\prime} \in \bar{T}$ s.t. $n_{T}^{\prime}=n$ occurs with $s$. This is well-defined because $\mathbf{T}$ is a join semi-lattice. In particular, for any $T \in \mathbf{T}$ and $n \in T$ there is a node $n^{\prime} \in \bar{T}$ such that $n_{T}^{\prime}=n$.

We say that information set $h_{i} \in H_{i}$ occurs with strategy profile $s \in S$ if some node $n \in \mathbf{D}_{i}$ with $h_{i}(n)=h_{i}$ occurs with $s$. Note that for this definition we do not require $n \in h_{i}$.

Figure 6: Illustration of Occur vs. Reached

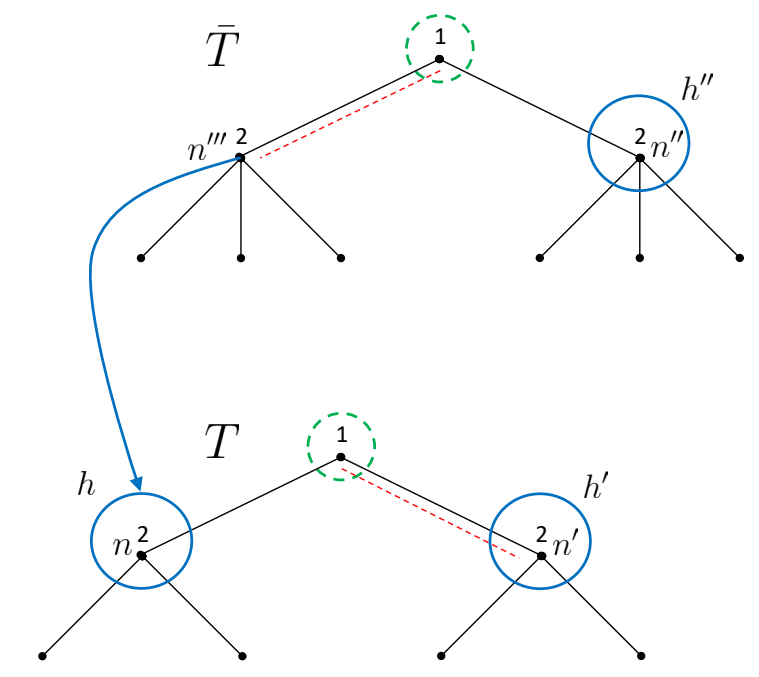

The following two examples will help to clarify the definition and its difference to the notion of a strategy reaching a node/information set. Consider first the example in Figure 6 . There are two trees, $\bar{T} \succ T$. There are two players, 1 and 2 . Player 1 moves first. If he moves left in tree $\bar{T}$, then player 2 remains unaware of her middle action. This is shown in Figure 6 by the blue arrow and disk (i.e., information set $h$ in $T$ ) upon player 1 moving left. Otherwise, if player 1 moves right in tree $\bar{T}$, player 2 becomes aware of middle (i.e., information set $h^{\prime \prime}$ ). (Player 1's initial information sets are indicated by disks with green intermitted boundaries.) Consider the strategy of player 1 indicated by the red intermitted edges. This strategy reaches only nodes $n^{\prime \prime \prime}$ and $n^{\prime}$. Yet, the nodes that occur with this strategy are $n^{\prime \prime \prime}$ and $n$. Thus, this example shows that the nodes reached may differ from nodes occurring. Note though that the nodes occurring with a strategy are not disjoint from the nodes reached with the strategy and that this intersection contains a node in the upmost tree $\bar{T}$. In terms of information sets, the 
strategy reaches only $h^{\prime}$ but the only information set occurring with this strategy is $h$. Thus, the example demonstrates that the information sets reached by a strategy may be even disjoint from the information sets occurring.

Figure 7: Another Illustration of Occur vs. Reached

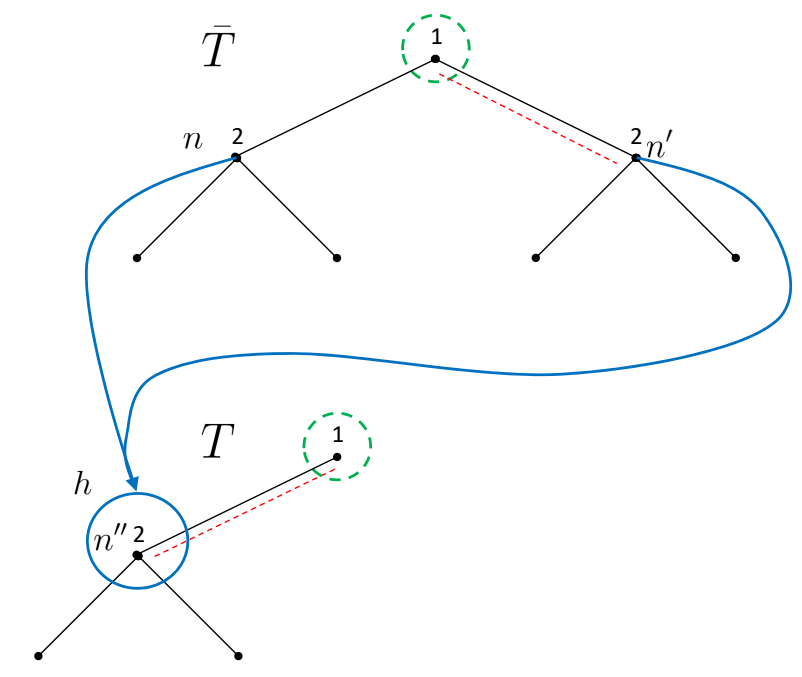

The example in Figure 6 has the feature that if an information set is reached (resp., occurs) with a strategy then also a node in this information set is reached (resp., occurs). With respect to the notion of occurring, this may not be the case in general as the next example shows. In Figure 7 there are also two trees, $\bar{T}$ and $T$. Obviously, tree $\bar{T}$ is more expressive than $T$ as it contains also the right action for player 1 (and all actions that follow). Player 2 is unaware of player 1's right action and remains unaware of it even if player 1 takes the right action. This is indicated by the blue information set belonging to player 2 in tree $T$. Even in the case in which player 1 chooses right and node $n^{\prime}$ occurs, player 2's information set is given by $h$. With respect to nodes, the strategy indicated by the red dashed line reaches only $n^{\prime}$ and $n^{\prime \prime}$. Yet, only $n^{\prime}$ occurs with this strategy. With respect to information set $h$, it is both reached by the strategy and occurs with the strategy. Note though that $h$ occurs with the strategy despite the fact that its only element, node $n^{\prime \prime}$ does not occur with the strategy. This is not a defect of the notion of a node/information set occurring but simply reflects the fact that in a game with unawareness the history occurring may not be in the occurring state of mind of a player. Note though that a player is not deluded either as she just misses an important fact rather than "making things up".

We summarize the examples:

Remark 2 In an extensive game with unawareness, if $s$ reaches $n$ then it is not necessarily the case that $n$ occurs with $s$. Similarly, if $n$ occurs with $s$ then it is not necessarily the case 
that $s$ reaches $n$. Moreover, information set $h$ may occur with a strategy $s$ even though $n$ with $h=\{n\}$ does not occur with $s$.

For the upmost tree $\bar{T}$ the following observation follows directly from the definitions:

Remark 3 Consider an extensive game with unawareness with the upmost tree $\bar{T}$. A strategy profile $s$ reaches $n \in \bar{T}$ if and only if $n$ occurs with $s$.

The observation means that the notions of node reached and node occurring with a strategy really depend on how these notions apply to less expressive trees $T \prec \bar{T}$. The notion of "reached" invokes the $T$-partial strategies to determined which node in $T$ is reached and is thus a more subjective notion (from the point of view of a player who considers tree $T$ and lower trees). The notion of "occur" invokes actions induced by the strategies in the highest possible tree. Thus it models the "actual" or "objective" play.

The following corollary follows now directly from the fact that any standard extensive game (i.e., without unawareness) just features one tree.

Corollary 1 In a standard extensive game (i.e., without unawareness), a strategy profile $s$ reaches $n$ if and only if $n$ occurs with $s$.

We say that node $n$ (resp., information set $h_{i}$ ) occurs with strategy $s_{i} \in S_{i}$ if there is a strategy profile $s_{-i} \in S_{-i}$ of the other players (and possibly nature) such that $n$ (resp., $h_{i}$ ) occurs with strategy profile $\left(s_{i}, s_{-i}\right)$. Analogously, we say that node $n$ (resp., information set $h_{i}$ ) occurs with strategy profile $s_{-i} \in S_{-i}$ if there exists a strategy $s_{i} \in S_{i}$ such that $n$ (resp., $\left.h_{i}\right)$ occurs with strategy profile $\left(s_{i}, s_{-i}\right)$.

We extend the definitions of information sets occurring to behavior and mixed strategies in the obvious way by considering nodes/information sets occurring with strict positive probability.

Let $N(s)$ denote the set of nodes in $\mathbf{N}$ that are reached with strategy profile $s$. Moreover, denote by $O(s)$ the set of nodes in $\mathbf{N}$ that occur with strategy profile $s$. We can now relate the notions of a node being reached and a node occurring with the following lemma:

Lemma 2 Consider an extensive games with unawareness. For any player $i \in I_{0}$ and strategies $s_{i}, s_{i}^{\prime} \in S_{i}$, if $N\left(s_{i}, s_{-i}\right)=N\left(s_{i}^{\prime}, s_{-i}\right)$ then $O\left(s_{i}, s_{-i}\right)=O\left(s_{i}^{\prime}, s_{-i}\right)$ for any $s_{-i} \in S_{-i}$. The converse does not necessarily hold.

Proof. For all $s_{-i} \in S_{-i}$, if $N\left(s_{i}, s_{-i}\right)=N\left(s_{i}^{\prime}, s_{-i}\right)$ then $N\left(s_{i}, s_{-i}\right) \cap \bar{T}=N\left(s_{i}^{\prime}, s_{-i}\right) \cap \bar{T}$. By Remark $3 O\left(s_{i}, s_{-i}\right) \cap \bar{T}=N\left(s_{i}, s_{-i}\right) \cap \bar{T}$ and $O\left(s_{i}^{\prime}, s_{-i}\right) \cap \bar{T}=N\left(s_{i}^{\prime}, s_{-i}\right) \cap \bar{T}$. Hence, $O\left(s_{i}, s_{-i}\right) \cap \bar{T}=O\left(s_{i}^{\prime}, s_{-i}\right) \cap \bar{T}$. By definition of node occurring, $O\left(s_{i}, s_{-i}\right)=O\left(s_{i}^{\prime}, s_{-i}\right)$. 
For the converse, we show a counterexample. Consider the extensive with unawareness in Figure 6. Further, let $s_{1}$ ascribe action "left" in tree $\bar{T}$ and "right" in tree $T$. Moreover, let $s_{1}^{\prime}$ ascribe action "left" both in tree $\bar{T}$ and $T$. Then information set $h$ occurs both with $s_{1}$ and $s_{1}^{\prime}$. In fact, $O\left(s_{1}, s_{-1}\right)=O\left(s_{1}^{\prime}, s_{-1}\right)$ for any $s_{1} \in S_{1}$. Yet, only strategy $s_{1}^{\prime}$ reaches $h$ in $T$ while $s_{1}$ reaches $h^{\prime}$ in $T$.

For any node $n$, any player $i \in I^{0}$, and any opponents' profile of strategies $s_{-i}$ (including nature if any), let $o\left(n \mid \beta_{i}, s_{-i}\right)$ and $o\left(n \mid \sigma_{i}, s_{-i}\right)$ denote the probability that node $n$ occurs with $\left(\beta_{i}, s_{-i}\right)$ and $\left(\sigma_{i}, s_{-i}\right)$, respectively.

Remark 4 In an extensive game with unawareness, it is not necessarily the case that for each tree $T \in \mathbf{T}, o\left(\cdot \mid \sigma_{i}, s_{-i}\right)$ defines a distribution over terminal nodes $Z \subseteq T$. E.g., in the example of Figure 7, no terminal node of $T$ occurs with the strategy of player 1 indicated by the red dashed line. Thus, o( $\left.\mid \sigma_{i}, s_{-i}\right)$ may not only be subadditive but may even assign zero to the set of all terminal histories in a given game tree. It is though a probability distribution over nodes in the upmost tree $\bar{T}$.

We use the notion of a node occurring to define another notion of equivalence between strategy that we dub realization-equivalent. For any player $i \in I^{0}$, a mixed strategy $\sigma_{i}$ and a behavior strategy $\beta_{i}$ are realization-equivalent if for every profile of opponents' strategies $s_{-i} \in$ $S_{-i}$ and every node $n \in \mathbf{N}$ of the extensive game with unawareness $o\left(n \mid \sigma_{i}, s_{-i}\right)=o\left(n \mid \beta_{i}, s_{-i}\right)$. Since information sets can be viewed as functions of nodes, realization-equivalent strategies are also realization-equivalent with respect to the probability of information sets occurring. This is relevant because information sets model also the player's state of mind. We like to assure that strategies are also equivalent with respect to the states of mind that may arise along the play.

Remark 5 If two strategies are realization-equivalent then also the same information sets occur with the same probabilities with both strategies.

Lemma 3 In any extensive game with unawareness and perfect recall, if $\mu_{i}$ and $\beta_{i}$ are equivalent to each other, then they are also realization-equivalent.

Proof. For any $\sigma_{i} \in \Delta\left(S_{i}\right), \beta_{i} \in B_{i}, s_{-i} \in S_{-i}, T \in \mathbf{T}, n \in T, o\left(n \mid \sigma_{i}, s_{-i}\right)=\rho\left(n^{\prime} \mid \sigma_{i}, s_{-i}\right)$ and $o\left(n \mid \beta_{i}, s_{-i}\right)=\rho\left(n^{\prime} \mid \beta_{i}, s_{-i}\right)$ for $n^{\prime} \in \bar{T}$ such that $\left(n^{\prime}\right)_{T}=n$. Let $\beta_{i}$ be equivalent to $\sigma_{i}$. Then the conclusion follows from Theorem 1 .

Theorem 1 and Lemma 3 now imply immediately the following corollary:

Corollary 2 In every extensive game with unawareness, if player $i$ has perfect recall, then for every mixed strategy of player $i$ there exists an realization-equivalent behavior strategy. 


\subsection{T-Partial Games and T-Partial Strategies}

We return to the fact that in extensive games with unawareness, strategies may only partially be an object of choice.

Let $S_{i}^{T}(n)$ be the set of all $T$-partial strategies of player $i$ that reach $n$. For this definition to make sense, note that $T_{n} \preceq T$. That is, if $s_{i} \in S_{i}^{T}(n)$ with $T \succeq T_{n}$ if and only if there exist $s_{-i} \in S_{-i}^{T}$ such that the profile $\left(s_{j}^{T_{n}}\left(h_{j}\left(n^{\prime}\right)\right)\right)_{j \in P\left(n^{\prime}\right)}$ in $n^{\prime} \in T_{n}$ leads to $n$.

Note that a $T$-partial game is an extensive game with unawareness in which the join of the join-semilattice of trees is $T$. Thus, Lemma 1 implies immediately

Corollary 3 Consider an extensive game with unawareness $\Gamma$. If $\Gamma$ satisfies perfect recall (i.e., I6), then for any player $i \in I, n \in N$ with $h_{i}(n) \in H_{i}$, and $n^{\prime} \in h_{i}(n), S_{i}^{T}(n)=S_{i}^{T}\left(n^{\prime}\right)$ for any $T \succeq T_{n}$.

This corollary is relevant because we view strategies of a player as objects of beliefs of other players. Yet, their beliefs are bounded by their awareness. That is, if player $i$ arrives at information set $h_{i}$, then his awareness level is given by $T_{h_{i}}$, the tree that contains information set $h_{i}$. Thus, he forms beliefs about player $j$ 's $T_{h_{i}}$-partial strategies.

Theorem 1 implies now immediately the version for $T$-partial strategies.

Corollary 4 In every extensive game with unawareness, if player $i$ has perfect recall, then for every T-partial mixed strategy of player $i$ there exists an equivalent $T$-partial behavior strategy, for $T \in \mathbf{T}$.

The original Kuhn Theorem is now a corollary for $T$ being a least expressive tree or $\mathbf{T}$ being singleton.

It is possible to also define a notion of node occurring with a $T$-partial strategy profile. Yet, such a definition is not very meaningful as the notion of a node occurring aims to characterize nodes that actually (or "objectively") occur. To determine such nodes, it is crucial consider which nodes are reached in the upmost tree $\bar{T}$. T-partial strategies, with $T \prec \bar{T}$, are by definition silent on it. Yet, every $T$-partial strategy can be extended to a strategy on the entire join-semilattice of trees T. Such an extension is typically not unique. The nodes occurring will then depend which extension is considered.

\section{References}

[1] Dubey, P. and M. Kaneko (1984). Information patterns and Nash equilibria in extensive games: I, Mathematical Social Sciences 8, 111-139. 
[2] Fagin, R. and J. Halpern (1988). Belief, awareness, and limited reasoning, Artificial Intelligence $34,39-76$.

[3] Feinberg, Y. (2012). Games with unawareness, mimeo.

[4] Filiz-Ozbay, E. (2012). Incorporating unawareness into contract theory, Games and Economic Behavior 76, 181-194.

[5] Grant, S. and J. Quiggin (2013). Inductive reasoning about unawareness, Economic Theory $54,717-755$.

[6] Halpern, J. and L.C. Rêgo (2014). Extensive games with possibly unaware players, Mathematical Social Sciences 70, 42-58.

[7] Heifetz, A., Meier, M., and B.C. Schipper (2013). Dynamic unawareness and rationalizable behavior, Games and Economic Behavior 81, 50-68.

[8] Heifetz, A., Meier, M., and B.C. Schipper (2012). Prudent rationalizability in generalized extensive-form games, University of California, Davis.

[9] Heifetz, A., Meier, M. and B.C. Schipper (2006). Interactive unawareness, Journal of Economic Theory 130, 78-94.

[10] Kuhn, H.W. (1953). Extensive games and the problem of information, in: Kuhn, H.W. and A.W. Tucker (eds.), Contributions to the Theory of Games, Vol. II, Annals of Mathematical Studies No. 28, Princeton University Press, Chapter 3, 193-216.

[11] Li, Y. and B.C. Schipper (2017). Disclosure under unawareness: An experiment, University of California, Davis.

[12] Maschler, M., Solan, E., and S. Zamir (2013). Game theory, Cambridge University Press.

[13] Ozbay, E. (2007). Unawareness and strategic announcements in games with uncertainty, in: Samet, D. (ed.), Proceedings of the 11th conference on Theoretical Aspects of Rationality and Knowledge,Presses Universitaires de Louvain, 231-238.

[14] Osborne, M. and A. Rubinstein (1994). A course in game theory, MIT Press.

[15] Piccione, M. and A. Rubinstein (1997). On the interpretation of decision problems with imperfect recall, Games and Economic Behavior 20, 3-24.

[16] Rêgo, L. and J. Halpern (2012). Generalized solution concepts in games with possibly unaware players, International Journal of Game Theory 41, 131-155.

[17] Schipper, B.C. (2017). Discovery and equilibrium in extensive games with unawareness, University of California, Davis. 
[18] Schipper, B.C. (2014). Unawareness - A gentle introduction to both the special issue and the literature, Mathematical Social Sciences 70, 1-9.

[19] Schipper, B.C. and H.Y. Woo (2017). Political awareness, microtargeting of voters, and negative electoral campaigning, University of California, Davis.

[20] Selten, R. (1975). Reexamination of the perfectness concept for equilibrium points in extensive games, International Journal of Game Theory 4, 25-55.

[21] Wichardt, P. (2008). Existence of Nash equilibria in finite extensive form games with imperfect recall: A counterexample, Games and Economic Behavior 63, 366-369. 